\title{
Frequência do aparecimento de dedo em gatilho no pós-operatório da síndrome do túnel do carpo em duas técnicas cirúrgicas: Aberta e endoscópica*
}

\section{Onset of Trigger Finger after Carpal Tunnel Syndrome Surgery: Assessment of Open and Endoscopic Techniques}

\author{
Marcela Fernandes ${ }^{1,2}$ João Carlos Belloti ${ }^{1,2}$ Aldo Okamura ${ }^{1,2}$ Jorge Raduan Neto ${ }^{1,2}$ \\ Rafael Tajiri²@ Flávio Faloppa1® Vinícius Ynoe de Moraes ${ }^{1-3(0)}$ \\ 1 Departamento de Ortopedia e Traumatologia, Universidade Federal \\ de São Paulo, São Paulo, SP, Brasil \\ 2 Serviço de Cirurgia da Mão, Hospital Alvorada, Moema, São Paulo, \\ SP, Brasil \\ Endereço para correspondência Vinícius Ynoe de Moraes, PhD, Rua \\ Borges Lagoa, 786, São Paulo, SP, 04041050, Brasil \\ (e-mail: vymoraes@gmail.com).
}

${ }^{3}$ Ortocity Serviços Médicos, São Paulo, SP, Brasil

Rev Bras Ortop 2021;56(3):346-350.

\section{Resumo \\ Palavras-chave \\ - síndrome do túnel do carpo \\ - parestesia \\ - estudo comparativo \\ - endoscopia \\ - dedo em gatilho}

Objetivo Determinar a frequência do aparecimento de dedo em gatilho (DG) no pósoperatório da síndrome do túnel do carpo (STC) em duas técnicas: aberta (TA) e endoscópica (TE). Como desfecho secundário, comparar as taxas de remissão da parestesia e dor residual entre as duas técnicas.

Métodos De forma prospectiva, verificamos o aparecimento de dedo em gatilho e taxa de remissão da parestesia e dor no território do nervo mediano em série de pacientes adultos operados pela TA $(n=34)$. Comparamos com coorte retrospectiva operada pela TE $(n=33)$, pela mesma equipe de cirurgiões. A avaliação dos pacientes ocorreu por meio de questionário estruturado em consulta de retorno, com mínimo de 6 meses de pós-operatório.

Resultados Sessenta e sete pacientes foram avaliados. Não houve diferença quanto ao aparecimento de dedo em gatilho (TA, $26,5 \%$ versus TE, $27,3 \% ; p=0,94$ ) e dor (TA, $76,5 \%$ versus TE, $84.8 \% ; p=0,38)$. Os pacientes operados pela TA apresentaram menos queixas de parestesia do que os operados pela TE (TA 5,9\% versus TE $24,2 \% ; p=0,03$ ). Conclusões Em nossa série, a técnica cirúrgica não influenciou o aparecimento de dedos em gatilho e dor residual. Os pacientes operados pela técnica aberta apresentaram menos queixa de parestesia residual pós-operatória.

\footnotetext{
Trabalho desenvolvido no Serviço de Residência Médica de Cirurgia e Microcirurgia da, Mão do Hospital Alvorada, São Paulo, SP, Brasil.
}

recebido 08 de Março de 2020 aceito 16 de Setembro de 2020
DOI https://doi.org/ $10.1055 / \mathrm{s}-0040-1721834$ ISSN 0102-3616.

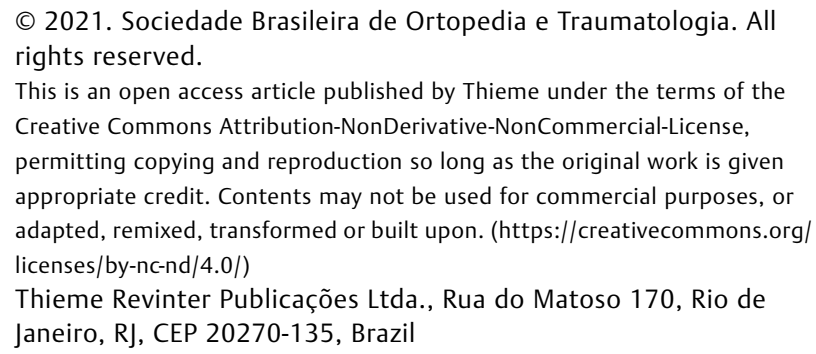

(c) 2021. Sociedade Brasileira de Ortopedia e Traumatologia. All rights reserved.

This is an open access article published by Thieme under the terms of the Creative Commons Attribution-NonDerivative-NonCommercial-License, permitting copying and reproduction so long as the original work is given appropriate credit. Contents may not be used for commercial purposes, or adapted, remixed, transformed or built upon. (https://creativecommons.org/ licenses/by-nc-nd/4.0/) Thieme Revinter Publicações Ltda., Rua do Matoso 170, Rio de Janeiro, RJ, CEP 20270-135, Brazil 
Abstract

\section{Keywords}

- carpal tunnel syndrome

- paresthesia

- comparative study

- endoscopy

- trigger finger disorder
Objective The present study aimed to determine the frequency of trigger finger (TF) onset after surgery for carpal tunnel syndrome (CTS) using an open (OT) or an endoscopic technique (ET). As a secondary endpoint, the present study also compared paresthesia remission and residual pain rates in patients submitted to both techniques. Methods Trigger finger onset and remission rates of paresthesia and pain at the median nerve territory was verified prospectively in a series of adult patients submitted to an OT procedure $(n=34)$. These findings were compared with a retrospective cohort submitted to ET $(n=33)$ by the same surgical team. Patients were evaluated with a structured questionnaire in a return visit at least 6 months after surgery.

Results Sixty-seven patients were evaluated. There was no difference regarding trigger finger onset (OT, 26.5\% versus ET, $27.3 \% ; p=0.94)$ and pain (OT, $76.5 \%$ versus ET, 84.8\%; $p=0.38)$. Patients submitted to OT had fewer paresthesia complaints compared with those operated using ET (OT, 5.9\% versus ET, 24.2\%; $p=0.03$ ).

Conclusions In our series, the surgical technique did not influence trigger finger onset and residual pain rates. Patients submitted to OT had less complaints of residual postoperative paresthesia.

\section{Introdução}

Define-se a síndrome do túnel do carpo (STC) como o conjunto de sinais e sintomas decorrentes da compressão do nervo mediano no nível do punho. ${ }^{1}$ Esta compressão pode estar associada, por exemplo, a tenossinovites ou à presença de estruturas anômalas dentro do túnel do carpo. ${ }^{2}$ A STC é descrita como a neuropatia compressiva mais prevalente, acometendo cerca de $2,7 \%$ da população geral, ${ }^{3}$ e com frequência aumentada em populações específicas, tais como diabéticos e trabalhadores braçais. ${ }^{3}$

O diagnóstico da STC é feito através da associação da história clínica, sintomatologia e manobras específicas. ${ }^{1}$ São sintomas comuns: dormência, parestesia, dor, perda de força na mão e no punho. Quando indicado, o tratamento cirúrgico se baseia na descompressão do túnel do carpo, por via aberta ou com auxílio de instrumentos de endoscopia. ${ }^{4-6}$

A tenossinovite estenosante dos tendões flexores dos dedos da mão - "dedo em gatilho" (DG) - é caracterizada por um processo inflamatório que acomete a bainha dos tendões flexores na região da primeira polia arciforme (A1). No dedo em gatilho, há espessamento da bainha e possível desenvolvimento de um nódulo no tendão flexor. Com isso, há consequente aumento do diâmetro do tendão, resultando em atrito entre a polia A1 e o tendão. ${ }^{7}$ Algumas doenças podem ser consideradas como predisponentes ao desenvolvimento do DG, tais como artrite reumatoide, diabetes, hipotireoidismo e amiloidose. ${ }^{8}$

No cenário do tratamento da STC e do DG, é frequente o aparecimento do DG após o tratamento cirúrgico da STC. Estudos reportam a frequência de 10 a 13\% em séries jordanianas, brasileiras e americanas. ${ }^{9-11}$ Neste interim, essa relação de causa-efeito tem sido discutida por diversos autores, ${ }^{9-12}$ mas a exata relação de ambas ainda não está bem esclarecida.

Dentre as opções de tratamento cirúrgico, pode-se realizar descompressão por via aberta tradicional, que consiste em acesso amplo à pele e fáscia palmar, ou por via endoscópica. ${ }^{2}$ É hipótese dos autores, corroborada por alguns estudos prévios, ${ }^{10,11}$ que a utilização de técnica minimamente invasiva, tal como a endoscópica, resulte em menor frequência de DGs, por preservar estruturas como a fáscia palmar e a pele imediatamente anterior ao ligamento transverso do carpo, o que, potencialmente, pode abrandar o efeito arco de corda resultante da descompressão do túnel do carpo. ${ }^{9-11}$

$O$ presente estudo tem como objetivo determinar a frequência de DG em pacientes que foram submetidos ao tratamento cirúrgico da STC, comparando duas técnicas de descompressão: aberta (TA) versus endoscópica (TE). Como desfecho secundário, verificaremos se há diferença nas taxas de remissão da parestesia e dor entre as duas técnicas avaliadas.

\section{Materiais e Métodos}

O presente estudo foi aprovado pelo comitê de ética em pesquisa, sob o número 17597019.7.0000.5533. Foi desenvolvido no serviço de residência médica de cirurgia da mão do Hospital Alvorada - Moema (São Paulo, SP) e da Clínica ortopédica Ortocity (São Paulo, SP). Trata-se de um estudo do tipo série de casos, com uma amostra prospectiva associada a coorte histórica retrospectiva, utilizada para comparação.

Foram incluídos pacientes adultos $>18$ anos, de ambos os sexos, que foram operados pela equipe de cirurgia de cirurgiões de mão (Moraes V. Y., Belloti J. C., Fernandes M., AO, Raduan Neto J.), em São Paulo, e que completaram no tempo mínimo de pósoperatório de 6 meses, pelas técnicas aberta ou endoscópica.

Não foram incluídos no estudo pacientes com outras doenças ou deformidades das mãos.

\section{Critérios de inclusão}

- Pacientes submetidos a tratamento cirúrgico da STC por técnica aberta ou endoscópica;

- Seguimento mínimo de 6 meses pós-operatório;

- Sem complicações intraoperatórias 
348 Frequência do aparecimento de dedo em gatilho no pós-operatório da STC Fernandes et al.

\section{Critérios de exclusão}

- Pacientes não concordantes para cooperar o com estudo;

- Pacientes com DG previamente ao procedimento cirúrgico para síndrome do túnel do carpo (diagnóstico clínico prévio, informado pelo paciente);

\section{Método de coleta de dados}

\section{Amostra de pacientes operados pela técnica aberta}

Trata-se de amostra de conveniência, resultante do ambulatório de especialidades. Estes pacientes foram tratados inicialmente por método conservador, com uso de corticosteroides e órtese noturna, por um período mínimo de 4 semanas. Na falha do tratamento conservador, os pacientes foram operados seguindo critérios de diagnóstico definidos, a saber: 1) presença de parestesia noturna no território do nervo mediano; 2) perda de capacidade de discriminação de dois pontos; 3 ) teste de Phalen positivo; 4) Sinal de Tinel positivo no nível do túnel do carpo; 5) parestesia no território do mediano; 6) perda de força na mão. Para a indicação do tratamento cirúrgico, esses pacientes deveriam apresentar ao menos três dos critérios expostos.

\section{Amostra de pacientes operados pela técnica endoscópica (controle retrospectivo)}

Realizamos o levantamento de pacientes operados pela técnica endoscópica com o auxílio dos registros dos controles cirúrgicos da equipe. Estes pacientes foram operados entre 2016 e 2019. Houve a identificação dos mesmos e coleta de dados demográficos: idade, gênero, profissão, mão dominante, doenças pré-existentes e dados relativos à doença/intervenção (Apêndice 1). Com estes dados, pareamos com os pacientes operados pela técnica aberta, a fim de tornarmos os grupos passíveis de comparação. A coleta de dados foi realizada preferencialmente em consulta presencial de retorno. Como alternativa, alguns contatos foram realizados por telefone com um questionário estruturado.

\section{Método de coleta das variáveis de interesse}

Para o diagnóstico de dedo em gatilho, realizamos as seguintes perguntas: 1) algum dos dedos apresenta-se travado ou com dificuldade de "dobrar" ou "esticar"?; 2) apresenta dor na "base" ou "raiz" dos dedos? Para a identificação de parestesia e dor realizamos as seguintes perguntas: 1) apresenta formigamento das mãos?; 2) apresenta algum desconforto nas mãos que atrapalha o sono?; 3) apresenta alguma dor ou desconforto nas mãos? Uma ou mais repostas "sim" foram consideradas como "eventos" para os objetivos do estudo.

\section{Análise Estatística}

$\mathrm{Na}$ análise estatística descritiva, expomos os dados contínuos como médias e seus respectivos desvios padrão(DP). Os dados categóricos foram expostos por seu número absoluto associados às suas porcentagens. Na análise estatística inferencial, optamos pela utilização de testes não-paramétricos. Utilizamos o teste U Mann-Whitney para variáveis contínuas e o teste $\mathrm{F}$ de Fisher para as variáveis categóricas. Consideramos $p<0,05$ para determinar diferenças entre grupos de comparação (significância estatística).
Tabela 1 Características das amostras e desfechos clínicos: Aberta e Endoscópica.

\begin{tabular}{|l|l|l|l|}
\hline & $\begin{array}{l}\text { Técnica } \\
\text { aberta }\end{array}$ & $\begin{array}{l}\text { Técnica } \\
\text { endoscópica }\end{array}$ & valor-p \\
\hline $\begin{array}{l}\text { Idade } \\
\text { (média, DP) }\end{array}$ & $58.7(3,5)$ & $52,2(3,7)$ & 0,02 \\
\hline $\begin{array}{l}\text { Sexo feminino } \\
\text { (n, \%) }\end{array}$ & $28(82,4)$ & $27(81,8)$ & 0,95 \\
\hline $\begin{array}{l}\text { Dominância, } \\
\text { direita (n, \%) }\end{array}$ & $30(88,2)$ & $28(84,8)$ & 0,68 \\
\hline $\begin{array}{l}\text { Comorbidades } \\
\text { (n, \%) }\end{array}$ & $21(61,8)$ & $14(42,4)$ & 0,11 \\
\hline $\begin{array}{l}\text { Presença de } \\
\text { diabetes (n, \%) }\end{array}$ & $9(26,5)$ & $8(24,2)$ & 0,83 \\
\hline $\begin{array}{l}\text { Presença de } \\
\text { dedo em } \\
\text { gatilho (n, \%) }\end{array}$ & $9(26,5)$ & $9(27,3)$ & 0,94 \\
\hline $\begin{array}{l}\text { Melhora da } \\
\text { parestesia (n, \%) }\end{array}$ & $32(94,1)$ & $25(75,8)$ & 0,03 \\
\hline $\begin{array}{l}\text { Melhora da } \\
\text { dor (n, \%) }\end{array}$ & $26(76,5)$ & $28(84,8)$ & 0,38 \\
\hline
\end{tabular}

Abreviação: DP, desvio padrão.

\section{Resultados}

\section{Caracterização dos grupos: técnica aberta versus endoscópica}

Incluímos 67 pacientes, todos com diagnóstico clínico compatível com STC. Trinta e três foram operados pela TE e 34 pela TA. As características demográficas dos grupos estão expostas na - Tabela 1.

\section{Variáveis pós-operatórias: presença de gatilho, melhora da dor e remissão da parestesia}

Os DGs ocorreram em $26,9 \%$ dos pacientes. Não houve diferença quanto à frequência do aparecimento de DG, quando agrupados pelas diferentes técnicas: TA, 26,5\% versus TE, $27,3 \% ; p=0,94$. Na avaliação da melhora da dor não houve diferença entre os grupos, TA, 76,5\% versus TE, $84.8 \%$; $p=0,38$. Na avaliação da melhora da parestesia, a TA demonstrou-se mais efetiva que a TE, $94.1 \%$ versus $75.8 \% ; p=0.03$.

\section{Discussão}

É sabido que pacientes com STC estão predispostos a desenvolver o DG, sendo sua incidência relatada em torno de 0,2 a 31,7\% por alguns estudos. ${ }^{9,11,13,14}$ Entretanto, estudos correlacionando o aparecimento do dedo em gatilho no pós-operatório do tratamento de STC são poucos. Um estudo ${ }^{11}$ que analisou 132 mãos operadas encontrou a frequência de $22 \%$ de DG. Hayashi et al., ${ }^{14}$ que realizaram outro estudo prospectivo com 164 mãos, encontraram uma frequência de DG de 31,7\%. Nossa série é condizente com o exposto na literatura, com frequência de aparecimento que variou de 26,5 a 27,3\%. Este achado fortalece a representatividade de nossa amostra, a despeito do relativo pequeno número amostral. Neste cenário, nos pareceu importante 
controlar as comorbidades, especialmente o diabetes, pois é consenso que há uma maior incidência de DG em diabéticos, identificada como o triplo da população sem a doença. ${ }^{15} \mathrm{Em}$ nossa série, os grupos se demonstraram comparáveis, no que diz respeito a frequência de comorbidades e diabetes, fato este que corrobora para a robustez de nossos resultados.Estudos ${ }^{10,11}$ sugerem que a liberação do túnel do carpo seria um fator de risco para o desenvolvimento de DGs, devido a alterações anatômicas e mecânicas. Com a liberação do ligamento carpal transverso, existe a hipótese que esta altera a biomecânica da ação dos flexores, aumentando seu "ângulo de ataque" distal, com consequente maior atrito no nível da entrada do túnel osteofibroso. Este evento pode contribuir para a gênese do DG ou incorrer na piora de um quadro prévio. Neste contexto, a liberação pela TE representaria menor dano tecidual: 1) pela menor via de acesso; 2) não-violação de estruturas anteriores ao LTC, tais como a pele e a fáscia palmar. Entretanto, nossos resultados não demonstraram a hipótese descrita, com frequência de dedos em gatilho bastante semelhantes entre os grupos.

Obtivemos diferença estatística no que diz respeito à melhora da parestesia entre os grupos, indicando melhor performance da TA. Em nosso estudo, apesar de robusta a diferença, devemos ter em mente que podemos entrar diante de viés de aferição, pois tratamos de coortes não simultâneas e com tempo de evolução de doença diferentes. Existem diversos estudos ${ }^{16-19}$ comparando as mesmas intervenções e desfechos. Nestes, os autores reportam taxa semelhante de melhora da parestesia ou da sensibilidade, com mensurações realizadas entre 6 e 12 meses de pós-operatório. Com população semelhante à nossa, o estudo de Okamura et al. ${ }^{20}$ reporta excelente e consistente resultado da TE para desfechos funcionais objetivos e o questionário de Boston. Apesar deste estudo incluir um número relevante de pacientes, ele carece de um grupo de comparação. Em outro desfecho relevante, dor, não identificamos benefício para qualquer das técnicas. Há de se pesar que parte da literatura refere benefício da TE, especialmente no primeiro mês. ${ }^{2}$

Algumas observações devem ser feitas sobre a validade interna $^{21}$ dos nossos resultados: 1) trata-se de amostra pequena, o que pode ter limitado a representatividade dos nossos dados; 2) característica retrospectiva, no que diz ao manejo inicial de diagnóstico e de tratamento conservador; 3) pela natureza do nosso estudo, não se pode imputar causaefeito, tão somente associação entre as duas doenças.

\section{Conclusão}

Nesta amostra, demonstramos que técnica cirúrgica parece não influenciar o aparecimento de DGs e de dor pós-operatória. Os pacientes operados pela TA apresentaram maior remissão da parestesia quando comparados aos operados pela técnica endoscópica.

\section{Suporte Financeiro}

Não houve suporte financeiro de fontes públicas, comerciais, ou sem fins lucrativos.

\section{Conflito de Interesses}

Os autores declaram não haver conflito de interesses.
Agradecimentos

Agradecimentos a Giovanna Arcaro de Lima, pelo suporte.

\section{Referências}

1 Chammas M, Boretto J, Burmann LM, Ramos RM, Santos Neto FC, Silva JB. Síndrome do túnel do carpo - Parte I (Anatomia, Fisiologia, Etiologia e Diagnóstico). Rev Bras Ortop 2014;49 (05):429-436

2 Chammas M, Boretto J, Burmann LM, Ramos RM, Santos Neto FC, Silva JB. Síndrome do túnel do Carpo - Parte II (Tratamento). Rev Bras Ortop 2014;49(05):437-445

3 Atroshi I, Gummesson C, Johnsson R, Ornstein E, Ranstam J, Rosén I. Prevalence of carpal tunnel syndrome in a general population. JAMA 1999;282(02):153-158

4 Verdugo RJ, Salinas RA, Castillo JL, Cea JG. Surgical versus nonsurgical treatment for carpal tunnel syndrome. Cochrane Database Syst Rev 2008;(04):CD001552

5 Okamura A, Guidetti BC, Caselli R, Borracini JA, Moraes VY, Belloti JC. How doboard-certified hand surgeons manage carpal tunnel syndrome? a national survey. Acta Ortop Bras 2018;26(01):48-53

6 Phalen GS. The carpal-tunnel syndrome. Seventeen years' experience in diagnosis and treatment of six hundred fifty-four hands. J Bone Joint Surg Am 1966;48(02):211-228

7 Ryzewicz M, Wolf JM. Trigger digits: principles, management, and complications. J Hand Surg Am 2006;31(01):135-146

8 Buch-Jaeger N, Foucher G, Ehrler S, Sammut D. The results of conservative management of trigger finger. A series of 169 patients. Ann Chir Main Memb Super 1992;11(03):189-193

9 El-Hadidi S. Is there a relation between carpal tunnel syndrome and trigger finger? BMC Proc 2015;9(Suppl 3):A65

10 Pires FA, Fernandes $\mathrm{CH}$, do Santos JBG. Does the presence of trigger finger affect the outcome of surgical treatment of carpal tunnel syndrome? A preliminary prognostic study. J Hand Surg Eur Vol 2018;43(09):999-1001

11 Zhang D, Collins J, Earp BE, Blazar P. Relationship of Carpal Tunnel Release and New Onset Trigger Finger. J Hand Surg Am 2019;44 (01):28-34

12 Assmus $\mathrm{H}$. [Tendovaginitis stenosans: a frequent complication of carpal tunnel syndrome]. Nervenarzt 2000;71(06):474-476

13 Shapiro S. Microsurgical carpal tunnel release. Neurosurgery 1995;37(01):66-70

14 Hayashi M, Uchiyama S, Toriumi H, Nakagawa H, Kamimura M, Miyasaka T. Carpal tunnel syndrome and development of trigger digit. J Clin Neurosci 2005;12(01):39-41

15 Fahey JJ, Bollinger JA. Trigger-finger in adults and children. J Bone Joint Surg Am 1954;36-A(06):1200-1218

16 Agee JM, McCarroll HR Jr, Tortosa RD, Berry DA, Szabo RM, Peimer CA. Endoscopic release of the carpal tunnel: a randomized prospective multicenter study. J Hand Surg Am 1992;17(06):987-995

17 Flores LP. [Endoscopic carpal tunnel release: a comparative study to the conventional open technique]. Arq Neuropsiquiatr 2005;63 (3A):637-642

18 Palmer DH, Paulson JC, Lane-Larsen CL, Peulen VK, Olson JD. Endoscopic carpal tunnel release: a comparison of two techniques with open release. Arthroscopy 1993;9(05):498-508

19 Urbaniak JR. Complications of treatment of carpal tunnel syndrome. In: Gelberman RH. Operative nerve repair and reconstruction. Philadelphia: Lippincott Williams \& Wilkins; 1991:967-979

20 Okamura A, Meirelles LM, Fernandes CH, Raduan Neto J, Dos Santos JB, Faloppa F. Evaluation of patients with carpal tunnel syndrome treated by endoscopic technique. Acta Ortop Bras 2014;22(01):29-33

21 de Moraes VY, Ferrari PM, Gracitelli GC, Faloppa F, Belloti JC. Outcomes in orthopedics and traumatology: translating research into practice. Acta Ortop Bras 2014;22(06):330-333 
350 Frequência do aparecimento de dedo em gatilho no pós-operatório da STC Fernandes et al.

Apêndice 1 Questionário do estudo - Túnel do carpo e aparecimento de dedo em gatilho

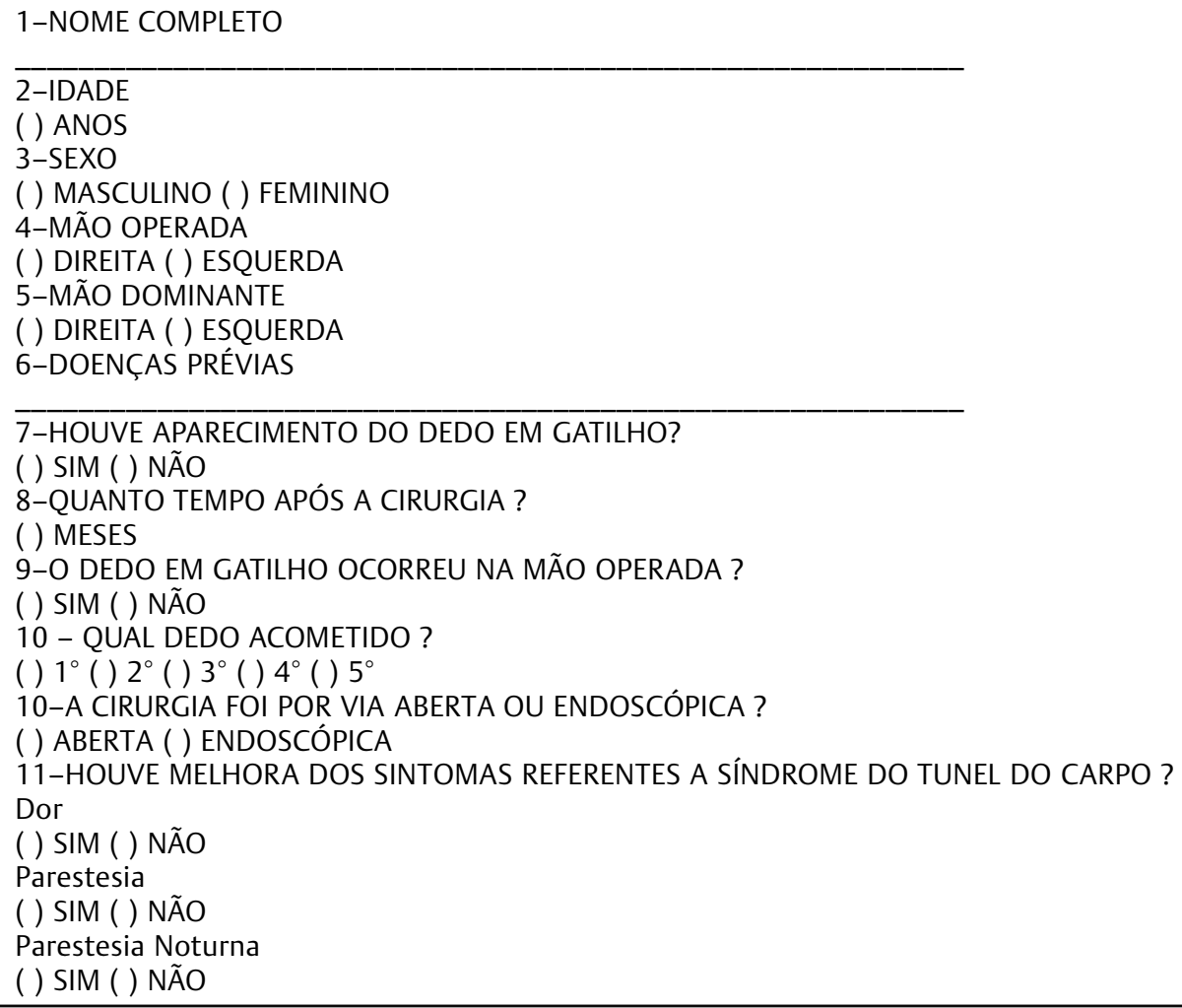

